\title{
ON CERTAIN ESTIMATIONS \\ FOR THE FIFTH AND SIXTH COEFFICIENT OF BOUNDED REAL UNIVALENT FUNCTIONS
}

\author{
RONALD KORTRAM and OLLI TAMMI
}

\section{Introduction}

The class of normalized bounded univalent functions $f$, defined in $U=$ $\{z \in C|| z \mid<1\}$, is

$$
S(b)=\left\{f\left|f(z)=b\left(z+a_{2} z^{2}+\ldots\right),\right| f(z) \mid<1,0<b \leqq 1\right\} .
$$

The wish to estimate the coefficients $a_{n}$ for lower indexes $n$ and at least for certain intervals of $b$ has led to a sequence of more or less successful attempts and methods. In a set of papers the authors have specialized in the estimation technique, applied mainly to $a_{4}$, which is based on an inequality obtainable by area integration and called here the Power inequality [2], [3], [4], [7]. In the real subclass $S_{R}(b) \subset S(b)$ the estimation of $a_{4}$ has recently been completed [1]. Therefore, testing the technique available for somewhat higher indexes is relevant just now.

In the estimations mentioned the knowledge of the lower coefficient bodies, denoted here by $\left(a_{2}, a_{3}\right),\left(a_{2}, a_{3}, a_{4}\right)$ etc., appeared to be of primary importance. The range of $a_{5}$ in $a_{2}, a_{3}, a_{4}$ was estimated by the aid of the Power inequality in [6]. Combining this with the knowledge of $\left(a_{2}, a_{3}, a_{4}\right)$ one can expect some improvement of the following result [3]: $a_{5}$ is maximized in $S(b)$ on $0.685 \leqq b \leqq 1$ by the mapping of the type $4: 4$ (we refer here to the notation $\alpha: \beta$ of the slit domains consisting of $U$ minus a collection of slits with $\alpha$ starting points and $\beta \geqq \alpha \geqq 1$ endpoints; [6], [7]). It appears that even in the class $S_{R}(b)$ the whole interval $e^{-2 / 3} \leqq b \leqq 1$, expected to be the best possible, cannot be reached. This clearly stresses what follows. Inequalities sharp for new types of algebraic equality function are needed. - The success in [1] was based on a process of this kind. Similarly, the importance and difficulty of governing the lower coefficient bodies increases with the index.

The above conclusions are confirmed further when the Power inequality for the coefficient $a_{6}$ in $S_{R}(b)$ is tested. 


\section{Maximizing $a_{5}$ in terms of $a_{2}$}

According to [6] we have for $a_{5}$ (the abbreviations $\Delta$ and $\gamma_{2}$ are taken from [6])

$$
\begin{gathered}
a_{5}-2 a_{2} a_{4}-\frac{3}{2} a_{3}^{2}+4 a_{2}^{2} a_{3}-\frac{3}{2} a_{2}^{4} \leqq \frac{1}{2}\left(1-b^{4}\right)-b^{2} a_{2}^{2} \\
-\left(\gamma_{2}+b^{2} a_{2}\right) \Delta^{-1}\left[\left(\gamma_{2}+b^{2} a_{2}\right) \log b-\left(a_{3}-\frac{1}{2} a_{2}^{2}\right) a_{2}\right] \\
-\left(a_{3}-\frac{1}{2} a_{2}^{2}\right) \Delta^{-1}\left[\left(a_{3}-\frac{1}{2} a_{2}^{2}\right)\left(a_{3}-a_{2}^{2}-1+b^{2}\right)-a_{2}\left(\gamma_{2}+b^{2} a_{2}\right)\right] ; \\
\gamma_{2}=a_{4}-2 a_{2} a_{3}+a_{2}^{3}, \\
\Delta=-\log b\left[a_{3}-1+b^{2}-\left(1+\frac{1}{\log b}\right) a_{2}^{2}\right]<0 .
\end{gathered}
$$

Rewrite this by the aid of a perfect square term which includes $a_{4}$ :

(2)

$$
\begin{gathered}
a_{5}-\frac{1}{2}\left(1-b^{4}\right) \leqq-\Delta^{-1} \log b\left(\gamma_{2}+b^{2} a_{2}-K\right)^{2}+\Delta^{-1} \log b K^{2} \\
-\Delta^{-1}\left(a_{3}-\frac{1}{2} a_{2}^{2}\right)^{2}\left(a_{3}-a_{2}^{2}-1+b^{2}\right)+\frac{3}{2} a_{3}^{2}-3 b^{2} a_{2}^{2}-\frac{1}{2} a_{2}^{4} \\
K=(\log b)^{-1} a_{2}\left(a_{3}-\frac{1}{2} a_{2}^{2}+\Delta\right) .
\end{gathered}
$$

Disregarding the limits of $a_{4}$ we maximize the right side in $\gamma_{2}$ :

$$
\begin{gathered}
a_{5}-\frac{1}{2}\left(1-b^{4}\right) \leqq A a_{3}^{2}+2 B a_{3}+C ; \\
A=\frac{3}{2}+\frac{1}{\log b}<0 \text { for } e^{-2 / 3}<b<1, \\
B=\left(\frac{1}{2 \log b}-\frac{1}{2}\right) a_{2}^{2}, \\
C=\left(1-4 b^{2}\right) a_{2}^{2}+\left(\frac{1}{2}+\frac{1}{4 \log b}\right) a_{2}^{4} .
\end{gathered}
$$

For $e^{-2 / 3}<b<1$, by maximizing the right side of (3) in terms of $a_{3}$, we are similarly led to the estimation

$$
a_{5}-\frac{1}{2}\left(1-b^{4}\right) \leqq a_{2}^{2}\left[1-4 b^{2}+\frac{1}{4} \frac{11+4 \log b}{2+3 \log b} a_{2}^{2}\right] .
$$

The equality here is reached on the parabola

$$
a_{3}=\frac{\log b-1}{3 \log b+2} a_{2}^{2} \text {. }
$$


The parabola (5) lies in the coefficient body $\left(a_{2}, a_{3}\right)$ (cf. e.g. [8], p. 49) so far as

$$
a_{2}^{2} \leqq\left(1-b^{2}\right) \frac{3 \log b+2}{2 \log b+3} .
$$

For the complementary values of $a_{2}$ the maximum of the right side of (3) is attained on the lower boundary arc $a_{3}=a_{2}^{2}-1+b^{2}$ of $\left(a_{2}, a_{3}\right)$.

Numerical evaluation yields the interval $0.66 \leqq b \leqq 1$ for which the right side of (3) is non-positive. Thus on this interval

$$
a_{4} \leqq \frac{1}{2}\left(1-b^{4}\right),
$$

and the equality is reached, as before, by the radial slit mapping $4: 4$.

\section{The use of $\left(a_{2}, a_{3}, a_{4}\right)$}

Some improvement can be expected when the limits of $a_{4}$ in terms of $a_{2}$ and $a_{3}$ are taken into consideration. From [7], p. 285, we read out for $\gamma_{2}$ :

$$
\begin{gathered}
-\frac{2}{3}\left(1-b^{3}\right)+\frac{b}{2} a_{2}^{2}-\frac{a_{2}^{3}}{12}+\frac{\left(a_{3}-\frac{3}{4} a_{2}^{2}-b a_{2}\right)^{2}}{2(1-b)+a_{2}} \leqq \gamma_{2} \\
\leqq \frac{2}{3}\left(1-b^{3}\right)-\frac{b}{2} a_{2}^{2}-\frac{a_{2}^{3}}{12}-\frac{\left(a_{3}-\frac{3}{4} a_{2}^{2}+b a_{2}\right)^{2}}{2(1-b)-a_{2}} .
\end{gathered}
$$

Applying this to evaluating the maximum of the right side of (2) in $\left(a_{2}, a_{3}\right)$ we can push the estimation (6) to the interval

$$
0.625 \leqq b \leqq 1 .
$$

The expected endpoint $e^{-2 / 3}=0.51$ is clearly outside the scope of the Power inequality. A substantial improvement necessitates an inequality sharp for the algebraic extremal functions of the more specific type $3: 4$ or $2: 4$.

\section{On the coefficient $a_{6}$}

Let us start from the general form of the Power inequality in $S_{R}(b)$ ([7], (6), p. $\left.177, x_{0}=0\right)$ :

$$
\sum_{-N}^{N} k y_{k}^{2} \leqq \sum_{-N}^{N} k x_{k}^{2} .
$$

Apply this, instead of $f(z)$, to $\sqrt{f\left(z^{2}\right)}$ and choose $N=5$. The numbers $y_{v}$ $(v=-5, \ldots, 5)$ are polynomials of the $a_{v}$-coefficients which include the parameters $x_{v}(v=-5, \ldots, 5)$. Introduce the new parameters $u_{k}=-k y_{-k}(k=1, \ldots, 5)$. From 
the lower cases we know to expect a maximizing inequality if the parameters are chosen to satisfy $y_{v}=-y_{-v}, x_{v}=-x_{-v}$. Moreover, for the even coefficient $a_{6}$ we may choose $u_{2}=u_{4}=0, u_{5}=1$. This leaves us the inequality

$$
\sum_{1}^{5} u_{v} y_{v} \leqq \sum_{1}^{5} \frac{\left|u_{v}\right|^{2}}{v} \quad\left(u_{2}=u_{4}=0\right)
$$

with

$$
\begin{gathered}
y_{1}=\frac{1}{2} a_{4}-\frac{3}{4} a_{2} a_{3}+\frac{5}{16} a_{2}^{3}+u_{3}\left(\frac{1}{2} a_{3}-\frac{3}{8} a_{2}^{2}\right)+\frac{1}{2} u_{1} a_{2}+x_{1} b^{1 / 2}, \\
y_{3}=\frac{1}{2} a_{5}-a_{2} a_{4}-\frac{5}{8} a_{3}^{2}+\frac{29}{16} a_{2}^{2} a_{3}-\frac{85}{128} a_{2}^{4}+u_{3}\left(\frac{1}{2} a_{4}-a_{2} a_{3}+\frac{13}{24} a_{2}^{3}\right) \\
+u_{1}\left(\frac{1}{2} a_{3}-\frac{3}{8} a_{2}^{2}\right)+\frac{1}{2} x_{1} b^{1 / 2} a_{2}+x_{3} b^{3 / 2}, \\
y_{5}=\frac{1}{2} a_{6}-a_{2} a_{5}-\frac{3}{2} a_{3} a_{4}+2 a_{2}^{2} a_{4}+\frac{21}{8} a_{2} a_{3}^{2}-\frac{59}{16} a_{2}^{3} a_{3}+\frac{689}{640} a_{2} \\
+u_{3}\left(\frac{1}{2} a_{5}-a_{2} a_{4}-\frac{5}{8} a_{3}^{2}+\frac{29}{16} a_{2}^{2} a_{3}-\frac{85}{128} a_{2}^{4}\right)+u_{1}\left(\frac{1}{2} a_{4}-\frac{3}{4} a_{2} a_{3}+\frac{5}{16} a_{2}^{3}\right) \\
+x_{1} b^{1 / 2}\left(\frac{1}{2} a_{3}-\frac{1}{8} a_{2}^{2}\right)+\frac{3}{2} x_{2} b^{3 / 2} a_{2}+x_{5} b^{5 / 2} \\
x_{1}=\left(2 a_{3}-\frac{1}{8} a_{2}^{2}\right) b^{1 / 2}, \\
x_{5}=\frac{1}{5} b^{5 / 2} . \\
x_{2} b^{3 / 2}, \\
x^{2}
\end{gathered}
$$

There are left two free parameters $u_{1}$ and $u_{3}$. From the experience gained from lower coefficients we know that the choice of $u_{1}$ and $u_{3}$ which eliminates $a_{4}$ and $a_{5}$ yields the maximum for $a_{6}$ in $a_{2}$ and $a_{3}$. Hence, take

and obtain for $a_{6}$

$$
u_{3}=a_{2}, \quad u_{1}=\frac{3}{2} a_{3}-\frac{a_{2}^{2}}{2}
$$

$$
a_{6}-\frac{2}{5}\left(1-b^{5}\right)
$$

$$
\leqq a_{2}^{2}\left[\frac{2}{3}-\frac{25}{6} b^{3}+\left(\frac{1}{2}-\frac{b}{32}\right) a_{2}^{2}-\frac{917}{960} a_{2}^{3}+\left(\frac{7}{2} a_{2}+b-3\right) a_{3}\right]+\left(\frac{9}{2}-8 b-\frac{7}{2} a_{2}\right) x_{3}^{2} .
$$


Again, we have to estimate the right side under the restriction given by the coefficient body $\left(a_{2}, a_{3}\right)$.

To the point $\left(a_{2}, a_{3}\right)$ one can find a restriction which is simpler than that implied in the coefficient body. The coefficients $a_{v}$ are limited, according to [5], (50), so that

$$
b^{2} \sum_{1}^{N} v\left|a_{v}\right|^{2} \leqq 1-\frac{N}{1-b^{2 N}} b^{2(N+1)}\left|a_{N}\right|^{2} .
$$

Setting $N=6$ yields

$$
2\left|a_{2}\right|^{2}+3\left|a_{3}\right|^{2} \leqq b^{-2}-1-b^{-2} \frac{6\left|a_{6}\right|^{2}}{1-b^{12}} .
$$

According to the idea presented in [3] we may restrict ourselves to the cases $\left|a_{6}\right| \geqq$ $\frac{2}{5}\left(1-b^{5}\right)$, which implies for the point $\left(a_{2}, a_{3}\right)$ the general restriction in $S_{R}(b)$ :

$$
\begin{aligned}
& \frac{a_{2}^{2}}{p^{2}}+\frac{a_{3}^{2}}{q^{2}} \leqq 1 \\
& p^{2}=\frac{1}{2}\left[b^{-2}-1-b^{-2} \frac{24}{25} \frac{\left(1-b^{5}\right)^{2}}{1-b^{12}}\right] \\
& q^{2}=\frac{2}{3} p^{2}
\end{aligned}
$$

Under this condition the right side of (8) remains non-positive for

where thus

$$
0.774 \leqq b \leqq 1
$$

$$
a_{6} \leqq \frac{2}{5}(1-b)^{5}
$$

The equality is attained by the radial slit mapping $5: 5$.

\section{References}

[1] JokINEN, O.: On the use of Löwner identities for bounded univalent functions. - Ann. Acad.

Sci. Fenn. Ser. A I Math. Dissertationes 41, 1982, 1-52.

[2] Kortram, R., and O. TAMmi: On the first coefficient regions of bounded univalent functions. Ann. Acad. Sci. Fenn. Ser. A I Math. 592, 1974, 1-27.

[3] Kortram, R., and O. TAMMI: On the range of validity of certain inequalities of the fourth coefficient of a bounded univalent function. - Bull. Soc. Sci. Lett. Łódź, 24, 1, 1974, $1-6$.

[4] KoRTRAM, R., and O. TAMMI: On the second coefficient region of bounded univalent functions. - Ann. Acad. Sci. Fenn. Ser. A I Math. 1, 1975, 155-175. 
[5] SChIfFer, M., and O. TAMmI: A Green's inequality for the power matrix. - Ann. Acad. Sci. Fenn. Ser. A I Math. 501, 1971, 1-15.

[6] TAMm, O.: Inequalities for the fifth coefficient for bounded real univalent functions. - J. Analyse Math. 30, 1976, 481-497.

[7] Tаммі, O.: Extremum problems for bounded univalent functions. - Lecture Notes in Mathematics 646. Springer-Verlag, Berlin-Heidelberg-New York, 1978.

[8] ТАммI, O.: Extremum problems for bounded univalent functions II. - Ibidem 913, 1982.

University of Nijmegen

Institute of Mathematics

Nijmegen

The Netherlands
University of Helsinki

Department of Mathematics

SF-00100 Helsinki

Finland

Received 3 October 1983 